\title{
STATIONARY SOLUTIONS TO CUBIC NONLINEAR SCHRÖDINGER EQUATIONS WITH QUASI-PERIODIC BOUNDARY CONDITIONS
}

\author{
ANDREA SACCHETTI
}

\begin{abstract}
In this paper we give the quantization rules to determine the normalized stationary solutions to the cubic nonlinear Schrödinger equation with quasi-periodic conditions on a given interval. Similarly to what happen in the Floquet's theory for linear periodic operators, also in this case some kind of band functions there exist.
\end{abstract}

\section{INTRODUCTION}

Nonlinear one-dimensional Schrödinger equations with cubic nonlinearity (hereafter NLS)

$$
i \hbar \frac{\partial \psi}{\partial t}=-\frac{\hbar^{2}}{2 m} \frac{\partial^{2} \psi}{\partial x^{2}}+\alpha|\psi|^{2} \psi
$$

on a finite interval $I=[0, a]$, for some $a>0$ fixed, may be of physical interest in the study of Bose-Einstein condensates trapped in a circular wave-guide (see, e.g., [4, 12, 15, see also 9] for a review). Wide attentions to the NLS (1) on a finite interval have been given from a mathematical point of view, with particular emphasis to the analysis of the existence and stability of standing waves solutions of the form $\psi(x, t)=e^{-i \mu t / \hbar} \phi(x)$ under different boundary conditions [2, 10, 11, 14, 18, 19. In fact, the function $\phi(x)$ is a normalized solution to the cubic time independent NLS (hereafter' denotes the derivative $\frac{\partial}{\partial x}$ and, for sake of simplicity, we fix the units such that $\hbar=1$ and $2 m=1$ ):

$$
-\phi^{\prime \prime}+\alpha|\phi|^{2} \phi=\mu \phi,\|\phi\|_{L^{2}(I, d x)}=1,
$$

and the boundary conditions considered in the above mentioned papers are the following ones: periodic boundary conditions (i.e. $\phi(0)=\phi(a)$ and $\phi^{\prime}(0)=\phi^{\prime}(a)$ ), Dirichlet boundary conditions (i.e. $\phi(0)=\phi(a)=0$ ), Neumann boundary conditions (i.e. $\phi^{\prime}(0)=\phi^{\prime}(a)=0$ ) and $\sigma$-walls boundary conditions (i.e. $\phi^{\prime}(0)=\sigma \phi(0)$ and $\left.\phi^{\prime}(a)=-\sigma \phi(a)\right)$ where the walls are repulsive if $\sigma>0$ and attractive when $\sigma<0$. In a couple of seminal papers Carr, Clark and Reinhardt [6, 7] studied the stationary solutions to (2) on a torus, that is with periodic boundary conditions, both in the case of attractive and repulsive cubic nonlinearities, and a key ingredient in their analysis was the use of the fundamental solution to a cubic NLS expressed through elliptic functions (see also [17, 20]).

Date: March 9, 2020.

This work is partially supported by GNFM-INdAM and by the UniMoRe-FIM project "Modelli e metodi della Fisica Matematica". 
This paper is addressed to the study of the normalized stationary solutions $\phi(x)$ to equation (2) with quasi-periodic boundary conditions on the interval $I=[0, a]$

$$
\left\{\begin{array}{l}
\phi(a)=e^{i k a} \phi(0) \\
\phi^{\prime}(a)=e^{i k a} \phi^{\prime}(0)
\end{array} \quad, k \in \mathbb{R} .\right.
$$

In the following, for argument's sake, we choose $a=1$.

Similarly to what happens in the Floquet's theory for linear periodic operators [13, even in this case we expect that it is possible to obtain an implicit relationship between the "energy" $\mu$ associated to the stationary solution and the "quasimomentum" variable $k$ that characterizes the quasi-periodic boundary conditions. Eventually, some analogies between the NLS equation (2) with quasi-periodic boundary conditions (3) and the Floquet's theory occur; for instance, in additions to plane wave solutions associated to the "energy" $\mu=k^{2}+\alpha$, other quasi-periodic solutions there exists for some values of the energy $\mu \in\left[\mu^{m}, \mu^{M}\right]$ and of the quasimomentum $k \in\left[k^{m}, k^{M}\right]$. The intervals $\left[\mu^{m}, \mu^{M}\right]$ and $\left[k^{m}, k^{M}\right]$ will depend on $\alpha$ and their amplitude is not zero when $\alpha \neq 0$. Finally, we give the algorithm for the computation of $k=k(\mu)$, when the "energy" $\mu$ belongs to the "energy band" $\left[\mu^{m}, \mu^{M}\right]$, and the numerical inversion of such a relation gives the "dispersion relation" $\mu=\mu(k)$. The names "energy band", "quasimomentum", "dispersion relation", etc., are adopted by the Floquet's theory.

The paper is organized as follows. In $₫ 2$ we collect some preliminary remarks. In $\$ 3$ we give the expression of the general solution to $(2)$ with quasi-periodic boundary conditions (3) and we compute the "energy band" $\left[\mu^{m}, \mu^{M}\right]$ and the associated interval $\left[k^{m}, k^{M}\right]$ of values for the "quasimomentum" $k$ to whom a normalized solution to (2) with boundary conditions (3) there exists. In particular, we also see that when the energy takes a value $\mu^{m}$ or $\mu^{M}$ at the edge of the energy band then we recover well known solutions. Finally, in Appendix A we collect some fundamental formulas concerning Jacobian elliptic functions.

\section{Preliminary REMARKS}

Remark 1. If $\phi(x)$ is a solution to (2) and (3) associated to an energy $\mu$ and to a quasimomentum $k$ then the complex conjugate $\overline{\phi(x)}$ is still a solution to (2) and (3) associated to the same energy $\mu$ and to the opposite quasimomentum $-k$. Therefore, we may restrict our attention to the case $k>0$.

Remark 2. We recall that if $\phi \in H^{2}(I)$ is a solution to the differential equation (2) when $I=\mathbb{R}$ then (see Lemma 3.7 [16]) $\phi$ is, up to a phase factor, a realvalued solution. We must remark that this regularity result does not hold true when $I=[0,1]$ is a finite interval and thus we actually may have complex-valued solutions to equation (2) with quasi-periodic boundary conditions (3).

Remark 3. Equation (2), with quasi-periodic boundary conditions (3), always admits plane wave solutions of the form $\phi(x)=e^{ \pm i \sqrt{\mu-\alpha} x}$ where $\mu=k^{2}+\alpha$.

Remark 4. When one looks for real valued solutions then equation (2) takes the form

$$
-\phi^{\prime \prime}+\alpha \phi^{3}=\mu \phi
$$


and it has a periodic solution (see Ch. 7, §10 [8], see also [1])

$$
\phi(x)=\frac{1}{\sqrt{\alpha}} t \sqrt{\frac{2 \mu}{1+t^{2}}} \operatorname{sn}\left(\left(x-x_{0}\right) \sqrt{\frac{\mu}{1+t^{2}}} ; t\right),
$$

where $\operatorname{sn}(x ; t)$ is an Jacobian elliptic function with parameters $x_{0} \in \mathbb{R}$ and $t \in[0,1)$ and real period $4 K(t)$, where $K(t)$ is the complete first elliptic integral. Making use of some formulas for $\operatorname{sn}(x ; t)$ one can gives other forms to the general solution; e.g., instead of (4) the general solution may be written as

$$
\phi(x)=\sqrt{\frac{-2 \mu t^{2}}{\alpha}} \operatorname{cn}\left(\left(x-x_{0}\right) \sqrt{\frac{\mu}{1-2 t^{2}}} ; t\right),
$$

or

$$
\phi(x)=\sqrt{\frac{2 \mu}{\alpha(2-t)}} \operatorname{dn}\left(\left(x-x_{0}\right) \sqrt{\frac{\mu}{t-2}} ; t\right),
$$

where $\operatorname{cn}(x ; t)$ and $\operatorname{dn}(x ; t)$ are Jacobian elliptic functions.

Remark 5. In the case of periodic boundary conditions then the solution has the form (4) where $\mu$ is given by

$$
\mu=16(n+1)^{2} K^{2}(t)\left(1+t^{2}\right), n=0,1,2, \ldots .
$$

On the other hand, in the case of out of phase boundary conditions, that is when $k=(2 n+1) \pi$, then the solution is still given by (4) where

$$
\mu=4(2 n+1)^{2} K^{2}(t)\left(1+t^{2}\right) .
$$

In both cases the value of the parameter $t$ must be such that the normalization condition holds true.

Remark 6. If $\phi(x)$ is a solution to equations (2) and (3) then $\phi_{x_{0}}(x)=\phi\left(x-x_{0}\right)$ is a solution to equations (2) and (3), too. Indeed, $u(x)=e^{-i k x} \phi(x)$ is a periodic function with period 1 and then

$$
e^{-i k x} \phi_{x_{0}}(x)=e^{-i k x_{0}} e^{-i k\left(x-x_{0}\right)} \phi\left(x-x_{0}\right)=e^{-i k x_{0}} u\left(x-x_{0}\right)
$$

is a periodic function with period 1, too.

\section{Solution to (2) with boundary conditions (3)}

3.1. Preliminaries. Following the approach proposed by [6, 7] we consider the Madelung transform $\phi(x)=\rho(x) e^{i \theta(x)}$, then equation (2) takes the form

$$
\left\{\begin{array}{l}
-\left(\rho^{\prime \prime}-\rho \theta^{2}\right)+\alpha \rho^{3}=\mu \rho \\
2 \rho^{\prime} \theta^{\prime}+\rho \theta^{\prime \prime}=0
\end{array} .\right.
$$

The second equation implies that $\rho^{2} \theta^{\prime}=C_{1}$, where $C_{1}=\rho_{0}^{2} \theta_{0}^{\prime}$ is a constant of integration.

Hereafter we assume, for argument's sake, that

$$
\theta_{0}=\theta(0)=0 \text {. }
$$

Remark 7. If $C_{1}=0$ then $\theta(t) \equiv \theta_{0}$ and $\phi$ is, up to a phase factor, a real valued solution already discussed in Remark 4; indeed, equation (7) reduces to $-\rho^{\prime \prime}+\alpha \rho^{3}=$ $\mu \rho$. 
Hereafter, we'll consider the case $C_{1} \neq 0$. In such a case $\rho(x)$ never takes zero values and

$$
\theta(x)=C_{1} \int_{0}^{x} \frac{1}{\rho^{2}(u)} d u, C_{1} \neq 0 .
$$

Because of the quasi periodic boundary conditions (3) it follows that $\rho(x)$ is a non negative solution to

$$
-\left(\rho^{\prime \prime}-\frac{C_{1}^{2}}{\rho^{3}}\right)+\alpha \rho^{3}=\mu \rho
$$

with periodic boundary conditions

$$
\rho(1)=\rho(0) \text { and } \rho^{\prime}(1)=\rho^{\prime}(0) .
$$

The two parameters $\mu$ and $C_{1}$ must satisfy to the condition $\theta(1)=k$, i.e.

$$
C_{1} \int_{0}^{1} \frac{1}{\rho^{2}\left(x ; C_{1}, \mu\right)} d x=k,
$$

because of (3), and to the normalization condition, that is

$$
\int_{0}^{1} \rho^{2}\left(x ; C_{1}, \mu\right) d x=1 .
$$

Remark 8. If one look for constant solutions to (9) then $\rho \equiv 1$, because of the normalization condition (12), $C_{1}=k$, because of (11), $\theta(x)=k x$ and equation (9) reduces to

$$
\frac{C_{1}^{2}}{\rho^{3}}+\alpha \rho^{3}=\mu \rho
$$

Therefore, since $\rho \equiv 1$, it follows that $\mu=k^{2}+\alpha$ and $\phi(x)=e^{i k x}$ is the plane wave solution already discussed in Remark 3 .

In order to look for non constant solutions we remark that equation $(9)$ can be solved by means of a simple squaring; indeed, if $\rho$ is not a constant function (we have already discussed this case in Remark 8$)$ then $(9)$ reduces to

$$
-\frac{1}{2} \rho^{\prime 2}-\frac{1}{2} \frac{C_{1}^{2}}{\rho^{2}}+\frac{1}{4} \alpha \rho^{4}-\frac{1}{2} \mu \rho^{2}=C_{2}
$$

where $C_{2}$ is a constant of integration. In we set $z=\rho^{2}$ then $z\left(x ; C_{1}, C_{2}, \mu\right)$ is a non negative solution to the equation

$$
z^{\prime 2}=f(z) \text { where } f(z)=b z^{3}+c z^{2}+d z+e
$$

with periodic boundary conditions $z(0)=z(1)$, where

$$
b=2 \alpha, c=-4 \mu, d=-8 C_{2} \text { and } e=-4 C_{1}^{2} .
$$

It is well known that the general solution to 15 has the form 8

$$
z(x)=A \operatorname{sn}^{2}\left(q x+x_{0} ; t\right)+B \text { with period } T_{\ell}=\frac{2 \ell K(t)}{q}, \ell=1,2, \ldots,
$$

for some $A, B, q, x_{0}$ and $t$, under the constraints

$$
C_{1}^{2}>0, B>0 \text { and } A>-B,
$$

because we assumed that $C_{1} \neq 0$ and that $z(x)$ never takes zero values. 
For argument's sake we can always assume that (see Remark 6)

$$
x_{0}=0
$$

by means of a translation argument $x \rightarrow x-x_{0} / q$.

Remark 9. In the following we restrict our attention to the case of $\ell=1$ in (16) and we denote by

$$
T:=T_{1}=\frac{2 K(t)}{q}
$$

the corresponding period of the solution $z(x)$. In such a way we'll obtain the first "band function" $\mu:=\mu_{1}=\mu_{1}(k)$. For different values of $\ell=2,3, \ldots$ we'll have the other "band functions" $\mu_{\ell}(k)$.

Remark 10. One can obtain a general solution to equation (15) even when $f(z)=$ $a z^{4}+b z^{3}+c z^{2}+d z+e$ is a fourth degree polynomial with $a \neq 0$; this case corresponds to the cubic/quintic NLS

$$
-\phi^{\prime \prime}+\alpha|\phi|^{2} \phi+\beta|\phi|^{4} \phi=\mu \phi,
$$

where $a=\frac{4}{3} \beta$. Indeed, let any $z_{0}>0$ be fixed; then it is known that when $f(z)$ is a quartic polynomial with non repeating factors then equation (15) has a general solution given by $z(x)=\zeta( \pm x)$ where

$$
\zeta(x)=z_{0}+\frac{\sqrt{f\left(z_{0}\right)} P^{\prime}(x)+\frac{1}{2} \dot{f}\left(z_{0}\right)\left[P(x)-\frac{1}{24} \ddot{f}\left(z_{0}\right)\right]+\frac{1}{24} f\left(z_{0}\right) f^{(3)}\left(z_{0}\right)}{2\left[P(x)-\frac{1}{24} \ddot{f}\left(z_{0}\right)\right]^{2}-\frac{1}{48} f\left(z_{0}\right) f^{(I V)}\left(z_{0}\right)}
$$

where $^{\prime}=\frac{d}{d x}$ denotes the derivative with respect to $x$ and ${ }^{\cdot}=\frac{d}{d z}$ denotes the derivative with respect to $z$, and where $P(x)=P\left(x ; g_{2}, g_{3}\right)$ is the Weierstrass's elliptic function with parameters

$$
g_{2}=a e-\frac{1}{4} b d+\frac{1}{12} c^{2} \text { and } g_{3}=-\frac{1}{16} e b^{2}+\frac{1}{6} e a c-\frac{1}{16} a d^{2}+\frac{1}{48} d b c-\frac{1}{216} c^{3} .
$$

This is an old and, as far as I know, almost unknown result due to Weierstrass. It was published in 1865, in an inaugural dissertation at Berlin, by Biermann [3], who ascribed it to Weierstrass; it was then mentioned in the book by Whittaker and Watson [21] in Ch. XX, Example 2, p.454. When $\beta=0$ then it is possible to prove that (17) reduces to (16).

Recalling 10 , that is the period $T$ of the solution $\rho(x)$ must be equal to 1 , the normalization condition

$$
1=\int_{0}^{1} z(x) d x=\int_{0}^{1}\left[A \operatorname{sn}^{2}(q x ; t)+B\right] d x=A F_{1}(t)+B
$$

where we set

$$
F_{1}(t):=\int_{0}^{1} \operatorname{sn}^{2}(q x ; t) d x
$$


and by substituting (16) in 15 and equating the coefficients of the same power of the function $\operatorname{sn}^{2}(q x ; t)$, it follows that

$$
\begin{aligned}
q & =2 K(t) \\
A & =\frac{1}{\alpha} 2 q^{2} t^{2}=\frac{8}{\alpha} K^{2}(t) t^{2} \\
B & =1-\frac{8 K^{2}(t) t^{2} F_{1}(t)}{\alpha} \\
\mu & =G(t)+\frac{3}{2} \alpha \\
C_{1}^{2} & =\frac{B}{4}(A+B)\left(2 \alpha B+4 q^{2}\right)
\end{aligned}
$$

where we set

$$
G(t):=4 K^{2}(t)\left[\left(1+t^{2}\right)-3 t^{2} F_{1}(t)\right] .
$$

Finally, the constant of integration $C_{2}$ is given by

$$
C_{2}=-\frac{1}{2} \alpha A B-B q^{2}-\frac{3}{4} \alpha B^{2}-\frac{1}{2} A q^{2} .
$$

Remark 11. We may remark that $G(t)$ is a monotone decreasing function such that

$$
\lim _{t \rightarrow 0} G(t)=4 K^{2}(0)=\pi^{2} \text { and } \lim _{t \rightarrow 1} G(t)=-\infty .
$$

For an explicit formula of the term $F_{1}(t)$ we refer to formula (33) in Appendix.

3.2. General solution. From (21) we obtain the equation $\mu=\mu(t)$, because of Remark 11 we may invert such an equation obtaining $t=t(\mu) \in[0,1)$ and finally $k=k(\mu)$; the inversion of such a latter relation will give the first (because we chose $\ell=1$, see Remark 9) "band function" $\mu(k)$.

We collect all these results in the following statement.

Theorem 1. Let $\mu \in \mathbb{R}$ and $\alpha \in \mathbb{R}, \alpha \neq 0$, be fixed. Let $q, A, B$ and $C_{1}$ given by (18), (19), 20) and 220). Let $t \in[0,1)$ be a solution to the following "quantization rule"

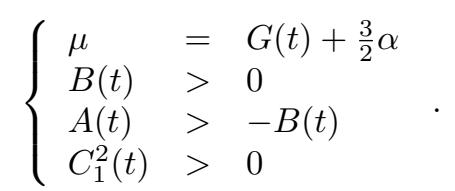

Then equation (2) with quasi-periodic boundary conditions (3) has a solution of the form $\phi(x)=\rho(x) e^{i \theta(x)}$ where $\rho(x)$ is a positive function given by

$$
\rho(x)=\sqrt{A \mathrm{sn}^{2}(q x ; t)+B}
$$

and where

$$
\theta(x)=C_{1} \int_{0}^{x} \frac{d u}{\rho^{2}(u)} .
$$

Theorem 1 provides some restrictions to the values allowed by energy $\mu$. Now, we are going to see how these constraints work in the case of attractive and repulsive nonlinearities. 
Theorem 2. For any $\alpha \in \mathbb{R}, \alpha \neq 0$, then 24) has just one solution $t \in[0,1)$ for any value $\mu \in\left(\mu^{m}, \mu^{M}\right)$ where

$$
\mu^{m}=G\left(t^{m}\right)+\frac{3}{2} \alpha \text { and } \mu^{M}=G\left(t^{M}\right)+\frac{3}{2} \alpha,
$$

and where $t^{m}$ and $t^{M}$ are given by (26) in the case of attractive nonlinearity $\alpha<0$, and by (28) in the case of repulsive nonlinearity $\alpha>0$.

Proof. Let us consider, at first, the case of attractive nonlinearity, i.e. $\alpha<0$. In such a case $B>0$ is always satisfied; furthermore condition $A>-B$ implies that

$$
A\left(1-F_{1}\right)>-1 \text { that is } 8 K^{2}(t) t^{2} F_{2}(t)+\alpha<0,
$$

where

$$
F_{2}(t):=1-F_{1}(t)=\int_{0}^{1} \mathrm{cn}^{2}(q x ; t) d x
$$

Condition $C_{1}^{2}>0$, under the constraints $B>0$ and $A+B>0$, becomes

$$
\left(2 \alpha B+4 q^{2}\right)=2 \alpha+16 K^{2}(t)\left(1-t^{2} F_{1}(t)\right)>0 .
$$

In conclusion, when $\alpha<0$ the quantization rule reads as

$$
\left\{\begin{array}{l}
\mu=G(t)+\frac{3}{2} \alpha \\
8 K^{2}(t) t^{2} F_{2}(t)+\alpha<0 \\
\alpha+8 K^{2}(t)\left(1-t^{2} F_{1}(t)\right)>0
\end{array} .\right.
$$

The two functions

$$
8 K^{2}(t) t^{2} F_{2}(t) \text { and } 8 K^{2}(t)\left(1-t^{2} F_{1}(t)\right)
$$

are monotone increasing functions such that

$$
\lim _{t \rightarrow 0} 8 K^{2}(t) t^{2} F_{2}(t)=0 \text { and } \lim _{t \rightarrow 1} 8 K^{2}(t) t^{2} F_{2}(t)=+\infty
$$

and

$$
\mathrm{L}:=\lim _{t \rightarrow 0} 8 K^{2}(t)\left(1-t^{2} F_{1}(t)\right)=2 \pi^{2}
$$

and

$$
\lim _{t \rightarrow 1} 8 K^{2}(t)\left(1-t^{2} F_{1}(t)\right)=+\infty
$$

Furthermore

$$
8 K^{2}(t)\left(1-t^{2} F_{1}(t)\right)-8 K^{2}(t) t^{2} F_{2}(t)=8 K^{2}(t)\left(1-t^{2}\right)>0, \forall t \in[0,1) .
$$

In conclusion: let $t_{1}$ be the unique solution to the equation

$$
8 K^{2}\left(t_{1}\right)\left(1-t_{1}^{2} F_{1}\left(t_{1}\right)\right)=-\alpha
$$

and let $t_{2}$ be the unique solution to the equation

$$
8 K^{2}\left(t_{2}\right) t_{2}^{2} F_{2}\left(t_{2}\right)=-\alpha
$$

Then,

$$
t^{M}=\left\{\begin{array}{ll}
0 & \text { if }-\mathrm{L} \leq \alpha<0 \\
t_{1} & \text { if } \alpha<-\mathrm{L}
\end{array} \text { and } t^{m}=t_{2}\right.
$$

proving thus Theorem 2 in the attractive case. 
We consider now the case of repulsive nonlinearity, i.e. $\alpha>0$. Then, condition $B>0$ implies that

$$
K^{2}(t) t^{2} F_{1}(t)<\frac{1}{8} \alpha
$$

Furthermore, condition $A>-B$ reduces to

$$
K^{2}(t) t^{2} F_{2}(t)>-\frac{1}{8} \alpha,
$$

which is always satisfied. Condition $C_{1}^{2}>0$ becomes

$$
\alpha+8 K^{2}(t)\left(1-t^{2} F_{1}(t)\right)>0
$$

which is always satisfied, too. In conclusion, when $\alpha>0$ the quantization rule reads as

$$
\left\{\begin{array}{l}
\mu=G(t)+\frac{3}{2} \alpha \\
\alpha-8 K^{2}(t) t^{2} F_{1}(t)>0
\end{array} .\right.
$$

We remark that the function $8 K^{2}(t) t^{2} F_{1}(t)$ is a monotone increasing function such that

$$
\lim _{t \rightarrow 0} 8 K^{2}(t) t^{2} F_{1}(t)=0 \text { and } \lim _{t \rightarrow 1} 8 K^{2}(t) t^{2} F_{1}(t)=+\infty .
$$

Let $t_{3}$ be the unique solution to the equation

$$
8 K^{2}\left(t_{3}\right) t_{3}^{2} F_{1}\left(t_{3}\right)=\alpha,
$$

then

$$
t^{M}=0 \text { and } t^{m}=t_{3}
$$

completing so the proof of the Theorem 2

The allowed values for the energy $\mu$ and for the quasimomentum $k$, as function of the nonlinearity parameter $\alpha$, are displaced in Figures 1 and 2 . In particular we plot the graph of the $\alpha$-dependent functions $\mu^{m}$ and $\mu^{M}$, and the graph of the functions

$$
k^{m}=\inf _{\mu \in\left(\mu^{m}, \mu^{M}\right)} k(\mu) \text { and } k^{M}=\sup _{\mu \in\left(\mu^{m}, \mu^{M}\right)} k(\mu) .
$$

There are three different behavior of the "band function" $\mu(k)$ and of the associated solutions (see Figure 3): when $\alpha<-\mathrm{L}$ then the band function is defined for any $k \in(0, \pi)$; when $-\mathrm{L}<\alpha<0$ then the band function is defined for any $k \in\left(k^{m}, \pi\right)$ where $k^{m} \in(0, \pi)$; when $0<\alpha$ then the band function is defined for any $k \in\left(\pi, k^{M}\right)$ where $k^{M}>\pi$. 


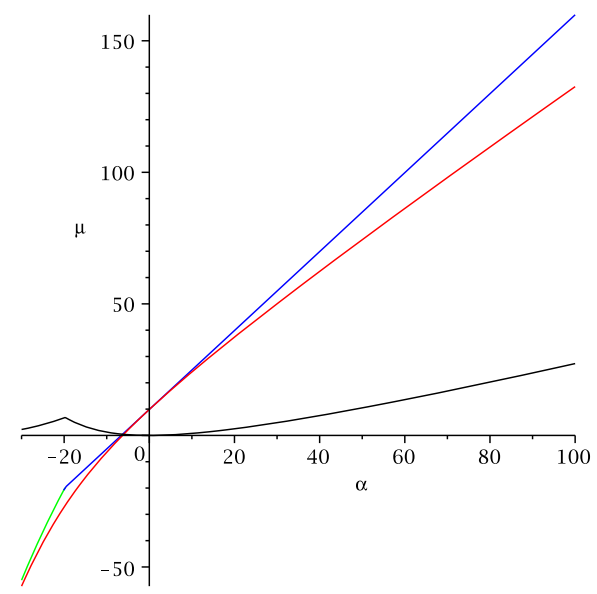

Figure 1. We consider the cubic model for $\alpha \in[-30,+100]$. We plot the graph of the functions $\mu^{M}(\alpha)$ (green line for $\alpha<-\mathrm{L}$ and blue line for $\alpha>-\mathrm{L}$ ) and $\mu^{m}(\alpha)$ (red line). The allowed values for the energy $\mu$ are the ones contained in the interval $\left(\mu^{m}, \mu^{M}\right)$. If we call $\mu^{M}-\mu^{m}$ the band width then it depends on $\alpha$ and it is zero only when $\alpha=0$ (black line). At the edges of the band $\left(\mu^{m}, \mu^{M}\right)$ we have that $C_{1}=0$ along the green line; $C_{1} \neq 0$ and $A=0$ and $B=1$ along the blue line; and finally $A=-B$ along the red line for $\alpha<0$ and $b=0$ along the red line for $\alpha>0$.

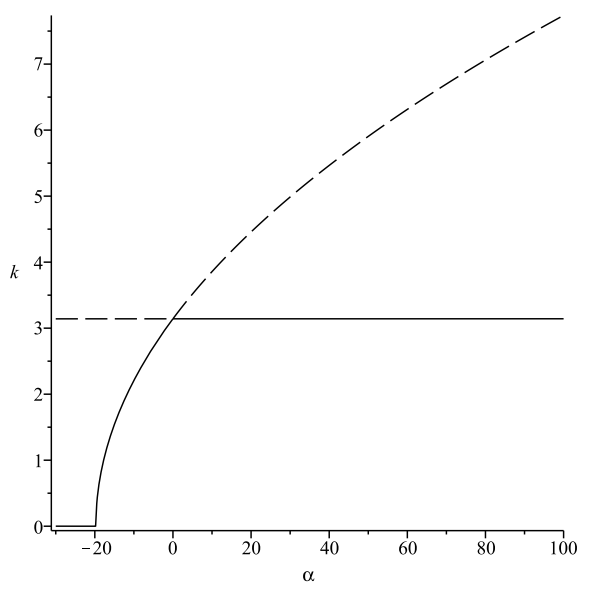

Figure 2. We consider the cubic model for $\alpha \in[-30,+100]$. We plot the graph of the functions $k^{m}$ (full line) and $k^{M}$ (broken line). As proved in Theorem $3 \lim _{\mu \rightarrow \mu^{m}} k(\mu)=\pi$.

3.3. Behavior of the solution at the boundaries $\mu^{m}$ and $\mu^{M}$. Here, we consider the behavior of the solution $\phi$ when $\mu$ takes the boundary values $\mu^{m}$ and $\mu^{M}$. 

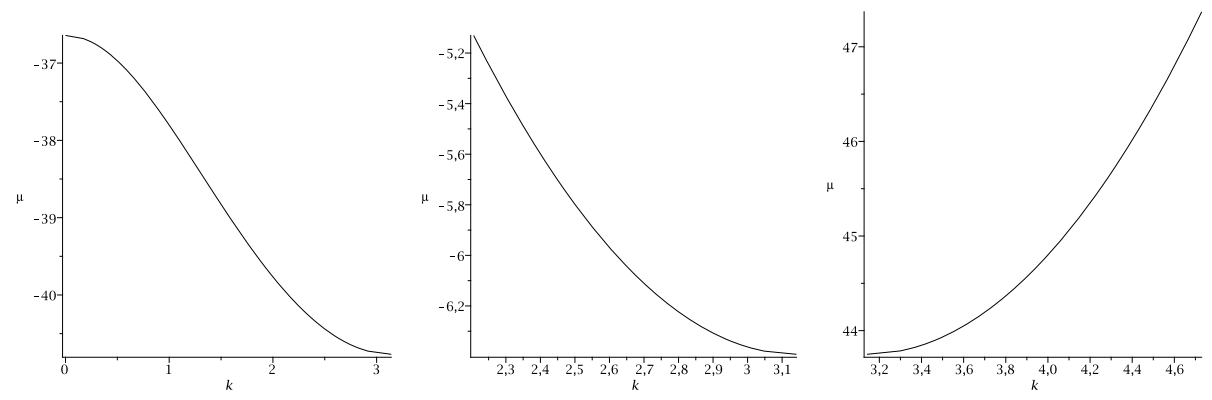

Figure 3. Here we plot the graph of the band functions $\mu(k)$ when $\alpha=-25$ (left hand side panel), $\alpha=-10$ (central side panel) and $\alpha=+25$ (right hand side panel).
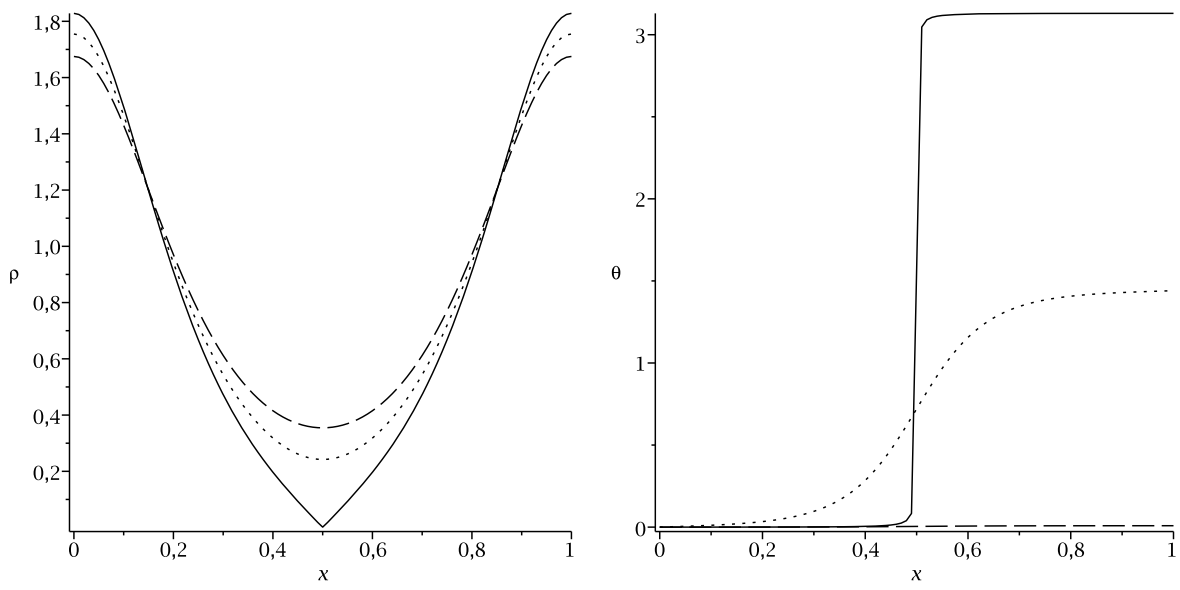

Figure 4. Here we plot the graph of the solution $\rho(x)$ (left hand side) and $\theta(x)$ (right hand side) when $\alpha=-25$. Broken lines correspond to the solutions associated to an energy $\mu$ close to the value $\mu^{M}$; full lines correspond to the solutions associated to an energy $\mu$ close to the value $\mu^{m}$; dot lines correspond to the solutions associated to the energy $\mu=\frac{1}{2}\left(\mu^{m}+\mu^{M}\right)$.

At first we consider the limit $\mu \rightarrow \mu^{M}$ where the proof of the Corollary below is an immediate conseguence of Theorem 2 .

Corollary 1. If $\alpha \geq-\mathrm{L}$, then $t^{M} \rightarrow 0, A \rightarrow 0, B \rightarrow 1$ and $k \rightarrow \sqrt{\alpha / 2+q^{2}(0)}$ as $\mu \rightarrow \mu^{M}=\pi^{2}+\frac{3}{2} \alpha$; in such a limit the solution is a plane wave function (see Figures 5 and 6, broken lines). If $\alpha<-\mathrm{L}$, then $t^{M} \neq 0$ and $C_{1} \rightarrow 0$ as $\mu \rightarrow \mu^{M}$; hence, in this limit we have that $\theta(x) \equiv 0$ and the solution has the form

$$
\phi(x)=C \operatorname{dn}(q x ; t)
$$

already discussed in (6) (see Figure 4. broken line). 

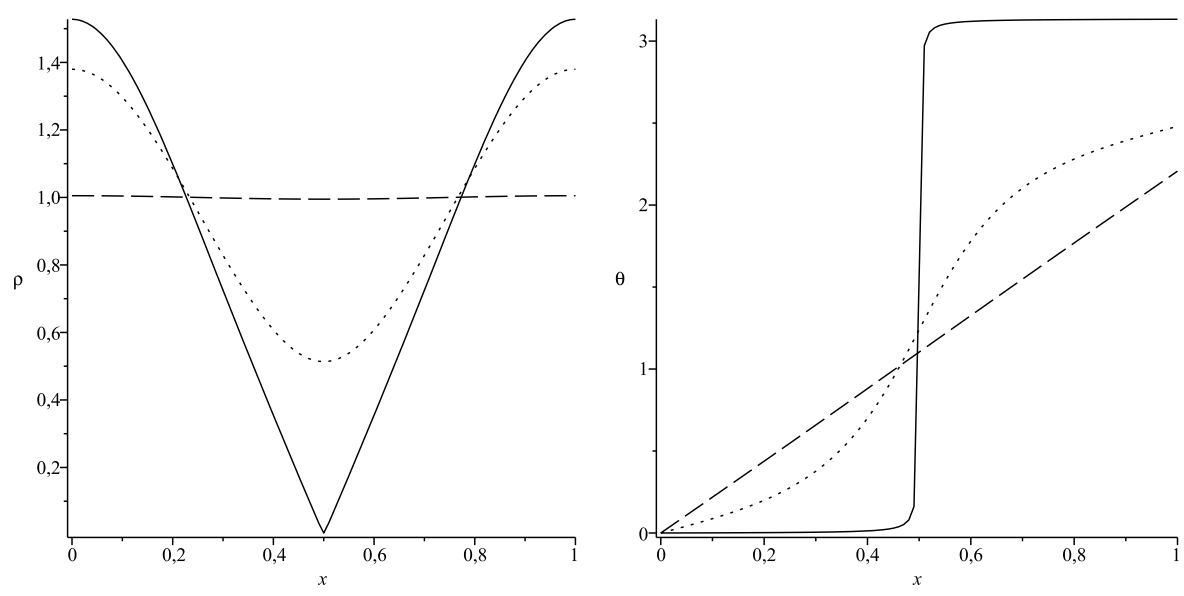

Figure 5. Here we plot the graph of the solution $\rho(x)$ (left hand side) and $\theta(x)$ (right hand side) when $\alpha=-10$. Full lines correspond to the solutions associated to an energy $\mu$ close to the value $\mu^{m}$; broken lines correspond to the solutions associated to an energy $\mu$ close to the value $\mu^{M}$; dot lines correspond to the solutions associated to the energy $\mu=\frac{1}{2}\left(\mu^{m}+\mu^{M}\right)$.
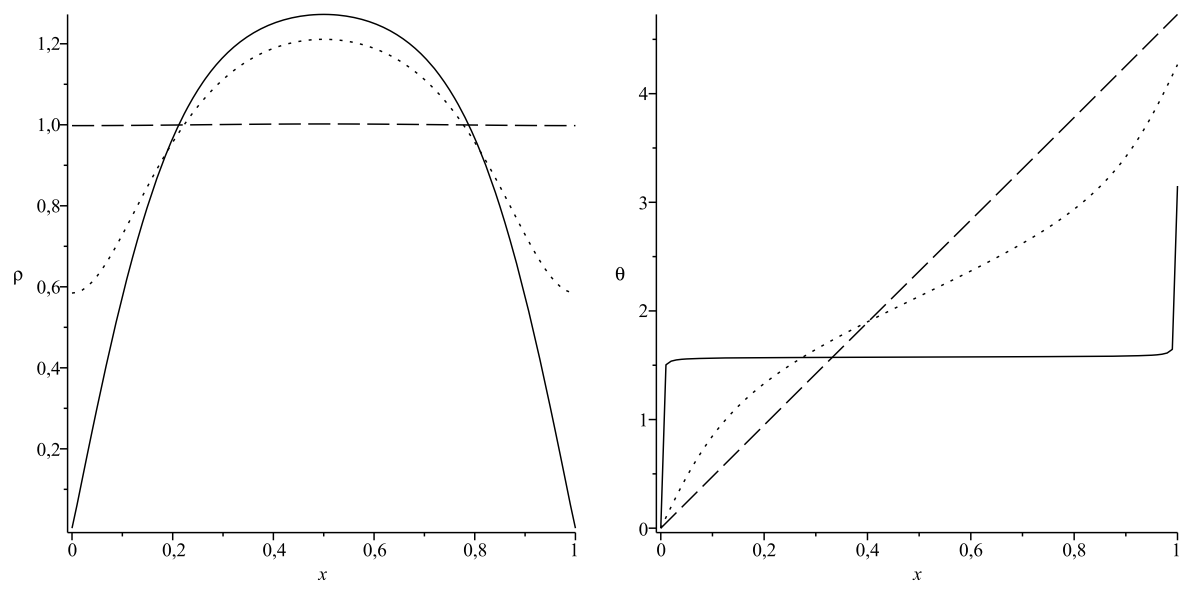

Figure 6 . Here we plot the graph of the solution $\rho(x)$ (left hand side) and $\theta(x)$ (right hand side) when $\alpha=+25$. Full lines correspond to the solutions associated to an energy $\mu$ close to the value $\mu^{m}$; broken lines correspond to the solutions associated to an energy $\mu$ close to the value $\mu^{M}$; dot lines correspond to the solutions associated to the energy $\mu=\frac{1}{2}\left(\mu^{m}+\mu^{M}\right)$.

We consider now the behavior of the solution when $\mu$ takes the limit value $\mu \rightarrow$ $\mu^{m}$. Even in this case the proof of the Corollary below is an immediate consequence of Theorem 2 . 
Corollary 2. If $\alpha<0$, then $A+B \rightarrow 0$ and $C_{1} \rightarrow 0$ as $\mu \rightarrow \mu^{m}$; in such a limit the solution has the form (see Figures 4 and 5 , full lines)

$$
\phi(x)=C \operatorname{cn}(q x ; t)
$$

already discussed in (5). If $\alpha>0$, then $B \rightarrow 0$ and $C_{1} \rightarrow 0$ as $\mu \rightarrow \mu^{m}$; in such a limit the solution has the form (see Figure 6 , full line)

$$
\phi(x)=C \operatorname{sn}(q x ; t)
$$

already discussed in (4).

Concerning the quasimomentum we recall that

$$
k^{m}=0 \text { if } \alpha \leq-\mathrm{L}
$$

and we can prove that

$$
k^{M}=\pi \text { if } \alpha<0 \text { and } k^{m}=\pi \text { if } \alpha>0 .
$$

\section{Theorem 3.}

$$
\lim _{\mu \rightarrow \mu^{m}} k(\mu)=\pi .
$$

Proof. In order to compute the quasimomentum $k(t)$ as function of the parameter $t$ we make use of equation (11) and we refer to the formula (34) in Appendix obtaining that

$$
\begin{aligned}
k & =C_{1} \int_{0}^{1} \frac{1}{A \operatorname{sn}^{2}(2 K(t) x ; t)+B} d x=2 C_{1} \int_{0}^{1 / 2} \frac{1}{A \operatorname{sn}^{2}(2 K(t) x ; t)+B} d x \\
& =\frac{\sqrt{(1+A / B)\left(2 \alpha B+16 K^{2}(t)\right)}}{2 K(t)} \Pi(1 ;-A / B, t)
\end{aligned}
$$

We consider then, at first, the attractive case $\alpha<0$; we have that

$$
k=\frac{\sqrt{2 \alpha+16 K(t) E(1 ; t)}}{2 K(t)} \sqrt{1-\zeta} \Pi(1 ; \zeta, t)
$$

where we set $\zeta=-A / B<1$ because of the constraints $A+B>0$ and $B>0$. Now, from Corollary 2 it follows that $A+B \rightarrow 0$ as $\mu \rightarrow \mu^{m}$ in the attractive case; then we have to compute the following limit

$$
\lim _{\zeta \rightarrow 1-0, t \rightarrow t_{2}} \sqrt{1-\zeta} \Pi(1 ; \zeta, t) .
$$

To this end we observe that $t_{2}<1$ and we recall that (see formula (412.01) [5])

$$
\sqrt{1-\zeta} \Pi(1 ; \zeta, t)=\sqrt{1-\zeta} K(t)+\frac{\pi \sqrt{\zeta}\left(1-\Lambda_{0}(\varphi, t)\right)}{2 \sqrt{\zeta-t^{2}}},
$$

when $t^{2}<\zeta<1$ and where $\varphi=\sin ^{-1}\left(\sqrt{(1-\zeta) / t^{\prime}}\right), t^{\prime}=\sqrt{1-t^{2}}$ and

$$
\Lambda_{0}(\varphi, t)=\frac{2}{\pi}\left[E(t) F\left(\varphi, t^{\prime}\right)+K(t) E\left(\varphi, t^{\prime}\right)-K(t) F\left(\varphi, t^{\prime}\right)\right],
$$

here $E(t)$ denotes the complete elliptic integral of the second kind with parameter $t$ and $F(\varphi, t)$ denotes the normal elliptic integral of first kind with argument $\varphi$ and parameter $t$. Hence,

$$
\lim _{\zeta \rightarrow 1-0, t \rightarrow t_{2}} \sqrt{1-\zeta} \Pi(1 ; \zeta, t)=\frac{\pi\left(1-\Lambda_{0}\left(0, t_{2}\right)\right)}{2 \sqrt{1-t_{2}^{2}}}=\frac{\pi}{2 \sqrt{1-t_{2}^{2}}}
$$


since $\varphi \rightarrow 0$ as $\zeta \rightarrow 1$. Hence, in such a limit we have that

$$
k\left(\mu^{m}\right)=\frac{\sqrt{2 \alpha+16 K\left(t_{2}\right) E\left(1 ; t_{2}\right)}}{2 K\left(t_{2}\right)} \cdot \frac{\pi}{2 \sqrt{1-t_{2}^{2}}}=\pi
$$

because $t_{2}$ is such that $8 K^{2}\left(t_{2}\right) t_{2}^{2} F_{2}\left(t_{2}\right)=-\alpha$.

In order to give the proof in the repulsive case $\alpha>0$ me still make use of formula (30) in the form

$$
k=\frac{\sqrt{2 \alpha B+16 K^{2}(t)}}{2 K(t)} \sqrt{1+\kappa} \Pi(1 ;-\kappa, t)
$$

where we set $\kappa=A / B>-1$. Now, from Corollary 2 it follows that $B \rightarrow 0$ as $\mu \rightarrow \mu^{m}$ in the repulsive case; then we have to compute the following limit for any $t<1$ fixed

$\lim _{\kappa \rightarrow+\infty} \sqrt{1+\kappa} \Pi(1 ;-\kappa, t)=\lim _{\kappa \rightarrow+\infty} \sqrt{1+\kappa} \int_{0}^{1} \frac{1}{\left(1+\kappa u^{2}\right) \sqrt{1-u^{2}} \sqrt{1-t^{2} u^{2}}} d u=\frac{1}{2} \pi$.

Hence, in such a limit we have that

$$
k\left(\mu^{m}\right)=\frac{\sqrt{2 \alpha+16 K\left(t_{3}\right) E\left(1 ; t_{3}\right)}}{2 K\left(t_{3}\right)} \cdot \frac{1}{2} \pi=\pi
$$

because $t_{3}$ is such that $8 K^{2}\left(t_{3}\right) t_{3}^{2} F_{1}\left(t_{3}\right)=\alpha$.

Remark 12. When $\alpha$ is small enough then $t^{M}=0$ and $\mu^{M}=\pi^{2}+\frac{3}{2} \alpha$. Furthermore, in the limit $\alpha \rightarrow 0$ a straightforward calculus gives that

$$
t_{2}:=t_{2}(\alpha) \sim \sqrt{-\frac{2 \alpha}{\pi}} \text { and } t_{3}:=t_{3}(\alpha) \sim \sqrt{\frac{2 \alpha}{\pi}} .
$$

In such a case the limit of the solution as $\alpha$ goes to zero becomes the plane wave solution discussed in Remark 3 associated to $k=\pi$.

\section{Appendix A. Some formulas concerning Jacobian Elliptic functions}

In order to give an explicit expression to the function $F_{1}(t)$ we recall that (see Formula 310.02 [5])

$$
F_{1}(t)=2 \int_{0}^{1 / 2} \operatorname{sn}^{2}(q x ; t) d x=\frac{q-2 E(\operatorname{sn}(q / 2 ; t) ; t)}{q t^{2}}=\frac{q-2 E(1 ; t)}{q t^{2}},
$$

since $\operatorname{sn}(q / 2 ; t)=1$ when $q=2 K(t)$, where $E(\varphi ; t)$ is the incomplete elliptic integral of second kind with argument $\varphi$ and parameter $t$.

Here, we collect some results concerning the elliptic integral of third kind defined as

$$
\Pi(z ; \nu, t)=\int_{0}^{z} \frac{1}{\left(1-\nu u^{2}\right) \sqrt{1-u^{2}} \sqrt{1-t^{2} u^{2}}} d u .
$$

Recalling that $\frac{d}{d x} \operatorname{sn}(x ; t)=\operatorname{cn}(x ; t) \operatorname{dn}(x ; t)$ and that $\sqrt{1-\operatorname{sn}^{2}(x ; t)}=|\operatorname{cn}(x ; t)|$ and $\sqrt{1-t^{2} \operatorname{sn}^{2}(x ; t)}=\operatorname{dn}(x ; t)$ then

$$
\int \frac{1}{A \operatorname{sn}^{2}(x ; t)+B} d x=\frac{1}{B} \Pi(\operatorname{sn}(x ; t) ;-A / B, t)
$$

provided that $x \in[0, K(t)]$ because $|\operatorname{cn}(x ; t)|=\operatorname{cn}(x ; t), B \neq 0,-A / B<1$ and $t \in[0,1)$. 


\section{REFERENCES}

[1] Abramowitz M, and Stegun I A, Handbook of Mathematical Functions with Formulas, Graphs, and Mathematical Tables, (New York: Dover) (1970).

[2] Angulo J, Non-linear stability of periodic traveling-wave equation for the Schrödinger and modified Korteweg-de Vries equation, J. of Differential Equations 235, 1 (2007).

[3] Biermann G G A, Problemata quaedam mechanica functionum ellipticarum ope soluta, Dissertation Inauguralis 1865 (Berlin).

[4] Brand J, and Reinhardt W P, Generating a ring currents, solitons, and svortices by stirring a Bose-Einstein condensate in a toroidal trap, J. Phys. B: At. Mol. Phys. 34, L113 2001.

[5] Byrd P F, and Friedman M D, Handbook of elliptic integrals for engineers and physicists, Springer-Verlag Berlin (1954).

[6] Carr L D, Clark C W, and Reinhardt W P, Stationary solutions of the one-dimensional nonlinear Schrödinger equation. I. Case of repulsive nonlinearity, Phys. Rev. A 62, 063610 (2000).

[7] Carr L D, Clark C W, and Reinhardt W P, Stationary solutions of the one-dimensional nonlinear Schrödinger equation. II. Case of attractive nonlinearity, Phys. Rev. A 62, 063611 (2000).

[8] Davis H T, Introduction to nonlinear differential and integral equations, Dover Publications (1962).

[9] Fibich G, The Nonlinear Schrödinger Equation: Singular Solutions and Optical Collapse, Springer Verlag (2016).

[10] Gallay T, and Hărăguş M, Stability of small periodic waves for the nonlinear Schrödinger equation, J. of Differential Equations 234, 544 (2007).

[11] Gallay T, and Hărăgus M, Orbital stability of periodic waves for the nonlinear Schrödinger equation, J. of Dyn. Diff. Eqns 19, 825 (2007).

[12] Gupta S, Murch K W, Moore K L, Purdy T P, and Stamper-Kurn D M, Bose-Einstein condensation in a circular waveguide, Phys. Rev. Lett. 95 (14):143201 (2005).

[13] Kohn W, Analytic properties of Bloch waves and Wannier functions, Physical Review 115 809 (1959).

[14] Landau L J, and Wilde I F, On the Bose-Einstein condensation of an ideal gas, Commun. Math. Phys. 70, 43 (1979).

[15] Morizot O, Colombe Y, Lorent V, Perrin H, and Garraway B M, Ring trap for ultracold atoms, Phys. Rev. A 74, 023617 (2006).

[16] Pelinovsky D E, Localization in periodic potentials; from Schrdinger operators to the GrossPitaevskii equation, London Mathematical Society, Lecture Note Series 390. Cambridge University Press, Cambridge (2011).

[17] Pérez-Obiol A, and Cheon T., Bose-Einstein condensate confined in a 1D ring stirred with a rotating delta link, preprint arXiv:1907.04574 (2019).

[18] Robinson D W, Bose-Einstein Condensation with Attractive Boundary Conditions, Commun. Math. Phys. 50, 53 (1976).

[19] Rowlands G, On the stability of solutions of nonlinear Schrödinger equation, IMA J. Appl. Math. 13, 367 (1974).

[20] Seaman B T, Carr L D, and Holland M J, Effect of a potential step or impurity on the Bose-Einstein condensate mean field, Phys. Rev. A 71033609 (2005).

[21] Whittaker E T and Watson G N, A Course of Modern Analysis (Cambridge: Cambridge University Press), 1927.

Department of Physics, Informatics and Mathematics, University of Modena and Reggio Emilia, Modena, Italy. 